\title{
Electrochemical and X-ray photoelectron spectroscopic investigations of conductive polymers
}

\author{
Umesh Somaji Waware $^{1} \cdot$ Ravi Arukula ${ }^{2}$ A. M. S. Hamouda ${ }^{1} \cdot$ Peter Kasak $^{3}$ \\ Received: 15 April 2019 / Revised: 26 August 2019 / Accepted: 3 September 2019 / Published online: 4 November 2019 \\ (C) The Author(s) 2019
}

\begin{abstract}
We report the synthesis of high soluble poly(aniline-co-o-hydroxyaniline) copolymers with varying the amount of ohydroxyaniline $(20,40,60$, and $80 \%)$ and referred as PA-co-o-HA20, PA-co-o-HA40, PA-co-o-HA60, and PA-co-o-HA80 respectively. The chemical and structural composition of the polymers and copolymers were determined by XPS, UV-Vis, and FE-SEM analysis. Electrochemical studies of the as-prepared polymers showed two single-electron oxidations and two singleelectron reductions reversibly at various scan rates ranging from 20 to $50 \mathrm{mV}$ and results reveals that the current density of the copolymer was lesser than the conventional polyaniline (PA). This is due to increasing the hydroxyl (-OH) branching on the polymer backbone in the polymer chain. The current density decreases from PA-co-o-HA20 to PA-co-o-HA80 by increasing the weight percentage of o-hydroxyaniline in the polymeric backbone.
\end{abstract}

Keywords Polyaniline $\cdot$ Poly(aniline-co-o-hydroxyaniline) $\cdot$ XPS and cyclicvoltametry

\section{Introduction}

Electrode materials play an important role in energy conversion and storage devices [1]. Conducting polymers, carbonbased, and metal compounds are amongst the three important kinds used as electrode materials for energy conversion/ storage devices. Conducting polymers ( $\mathrm{CPs})$, such as polypyrrole (PPy) and polyaniline (PA) and poly(3,4-ethylene dioxythiophene) (PEDOT), have immense attention in recent years because of their prospective uses in various fields such as supercapacitors, batteries, sensors, fuel cells, electrochromic devices, anticorrosive coatings, and electroactive polymers [2-10].

Electronic supplementary material The online version of this article (https://doi.org/10.1007/s11581-019-03250-8) contains supplementary material, which is available to authorized users.

Umesh Somaji Waware uswaware@gmail.com

1 Department of Mechanical and Industrial Engineering, Qatar University, P.O. Box 2713, Doha, Qatar

2 Department of Chemistry, GITAM University, Hyderabad, India

3 Center for Advanced Materials, Qatar University, P.O. Box 2713, Doha, Qatar
Amongst all conducting polymers, PA is a unique class material due to having low cost, easy preparation, commendable environmental stability, good electrical conductivity, and electrochemical activity $[11,12]$. But, due to low processability and intractable nature of acid-doped PA that restricts the few applications in industry [13]; to overcome this problem, several researchers have been extensively improved the field in terms of synthesis, processability, and various applications [14-16]. The more significant strategies to attaining the good processability for conducting polyaniline are (i) to familiarize substituent groups on the phenyl ring [17, 18], (ii) self-doping (- $\mathrm{SO}_{3} \mathrm{H}$ ring substituted) polyanilines $[19,20]$, and (iii) blending with conventional solvable polymers like poly(vinyl alcohol) [21]. As stated above, substituted polyanilines are soluble in common organic solvents [17]. Owing to distorted planarconjugation, the substituent conducting polyaniline attains the less conductivity. To improve the processability and conductivity in conducting polymers, the copolymers of aniline and substituted anilines have been introduced. Numerous aniline copolymers are well known from the literature [22, 23]. However, to the best of our knowledge, only a few studies report the extensive structural elucidation of conducting polyaniline copolymer.

The foremost studies on the structural interpretation of polyaniline or copolyanilines are mainly, optical absorption $[24,25]$, electron spin resonance [26], nuclear magnetic 
resonance [27], electrochemical activity [28] measurements, and Raman and IR studies [29-31] of polymers prepared under various conditions. The huge experimental and theoretical work have done on different forms of polyaniline has; however, not absolutely determined the molecular structure of the polymer and copolymers. However, MacDiarmid et al. [32] and Baughman et al. [33] were proposed a highly delocalized configuration structures of polyaniline, wherein the polyemeraldine salt (ES) is considered to be made up of equivalent nitrogen atoms, and the six-membered ring structure and the $\mathrm{C}-\mathrm{N}$ bonds are considered equivalent in all respects. Still, there is a challenge to extensive structural elucidation of conducting polyaniline copolymer using electronic studies like X-ray photoelectronic spectroscopy (XPS).

The intention of the present work is to investigate detailed cyclic voltammetry and XPS of PA, P-o-HA, and high soluble poly(aniline-co-o-hydroxyaniline) copolymers. The XPS is a dominant surface analysis tool extensively used in polymers; polymer blending and composites field [34] and this method reveals the composition, oxidation state, and chemical bonding of atoms found in a near-surface region of the thickness of 5 to $10 \mathrm{~nm}$. The chemical composition can be found based on the binding energies and peak intensity. The formal characterizations of PA, P-o-HA, and high soluble poly(aniline-co-ohydroxyaniline) copolymers, electrochemical, and XPS study were thoroughly investigated as follows.

\section{Experimental section}

\section{Chemicals and materials}

Aniline, o-hydroxyaniline, and ammonium persulfate were purchased from the Sigma-Aldrich and used as received. Analytical grade hydrochloric $(\mathrm{HCl})$ acid and spectroscopic grade dimethylformamide (DMF), (THF), $\mathrm{N}$-methylpyrrolidone (NMP), and methanol were used as received. Double-distilled (DW) water was used in the preparation of aqueous solutions.

\section{Synthesis}

\section{Synthesis of PA and P-o-HA}

Polyaniline (PA) and poly(o-hydroxyaniline) P-o-HA were synthesized by oxidation of aniline and $o$-hydroxyaniline separately with ammonium persulfate in $1 \mathrm{M}$ hydrochloric acid according to modified procedure reported [22]. In a typical reaction, aniline $(20 \mathrm{mmol}, 1.86 \mathrm{~g})$ was added to $200 \mathrm{~mL}$ of $1 \mathrm{M} \mathrm{HCl}$ and the mixture was cooled to $0-4{ }^{\circ} \mathrm{C}$ in an ice bath. An ice-cold solution of $20 \mathrm{mmol}(4.56 \mathrm{~g})$ ammonium persulfate dissolved in $200 \mathrm{ml}$ of $1 \mathrm{M} \mathrm{HCl}$ was then added dropwise into the monomer solution with constant stirring under nitrogen atmosphere at $0-4{ }^{\circ} \mathrm{C}$. The reaction mixture was further stirred for another $5 \mathrm{~h}$ and aged at room temperature for $24 \mathrm{~h}$ which resulted in a greenish precipitate of the polymer in emeraldine salt form (PA hydrochloride). It was filtered and washed with small volumes of $1 \mathrm{M} \mathrm{HCl}$ and dried in an air oven for $8 \mathrm{~h}$ at $80^{\circ} \mathrm{C}$. The base form of the polymer was obtained by treatment of emeraldine salt with $1 \mathrm{M} \mathrm{NH}_{4} \mathrm{OH}$ solution and kept for $24 \mathrm{~h}$ at room temperature. The separated base was washed with distilled water, filtered, and air-dried. Similarly, poly(o-hydroxyaniline), P-o-HA was obtained by oxidative polymerization of o-hydroxyaniline using ammonium persulfate. The final product of the polymer powder obtained was brown in color.

\section{Synthesis of copolymers (PA-co-o-HA)}

Copolymers of aniline and o-hydroxyaniline (PA-co-o-HA) were obtained by chemical oxidative coupling of the monomers in $1 \mathrm{M} \mathrm{HCl}$ using ammonium persulfate in a similar way. Different copolymers were prepared by varying the amount of o-hydroxyaniline, namely $20,40,60$, and $80 \%$. The copolymers with $20,40,60,80 \%$ o-hydroxyaniline will be referred from here on as PA-co-o-HA20, PA-co-o-HA 40, PA-co-oHA 60, and PA-co-o-HA 80 respectively. The structure and synthetic procedure of copolymers (PA-co-o-HA X) are shown in Scheme 1 and the 3D Chemical structures of PA, P-o-HA, and copolymers were shown in Scheme 2.

\section{Modification of glassy carbon electrode}

First, the working electrode, glassy carbon electrode (GCE) was polished with $1.0 \mu \mathrm{m}$ alumina slurry and sonicated in DW. The cleaned GCE was subsequently dried using a purified nitrogen stream. The casting solution was prepared in DMF solvent with a concentration of $5 \mathrm{mg} / \mathrm{mL}$. In order to obtain a homogeneous dispersion of the solution was

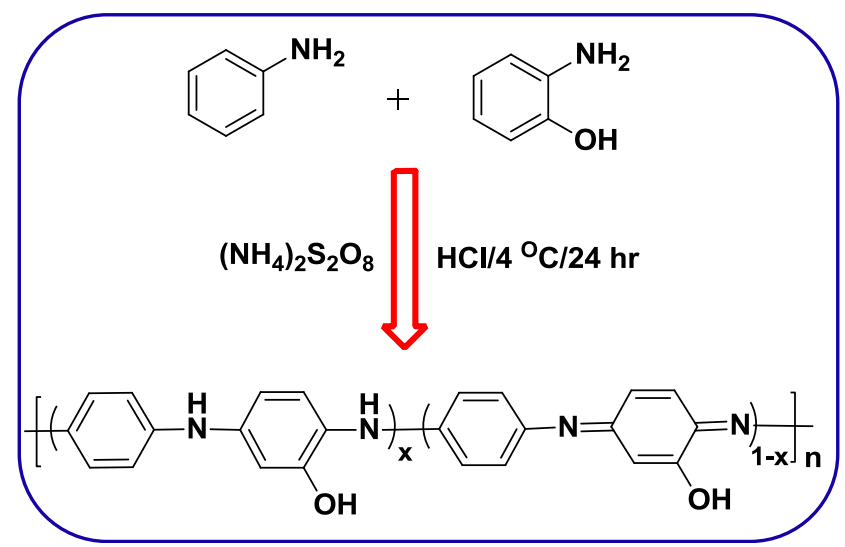

Scheme 1 Synthetic route for the preparation of HCl-doped polyaniline, poly(o-hydroxyaniline), and its various copolymers 
Scheme 2 3D chemical structures of a polyaniline PA, $\mathbf{b}$ poly(o-hydroxyaniline) P-o-HA, c poly(aniline-co-ohydroxyaniline) derivatives PAco-o-HA

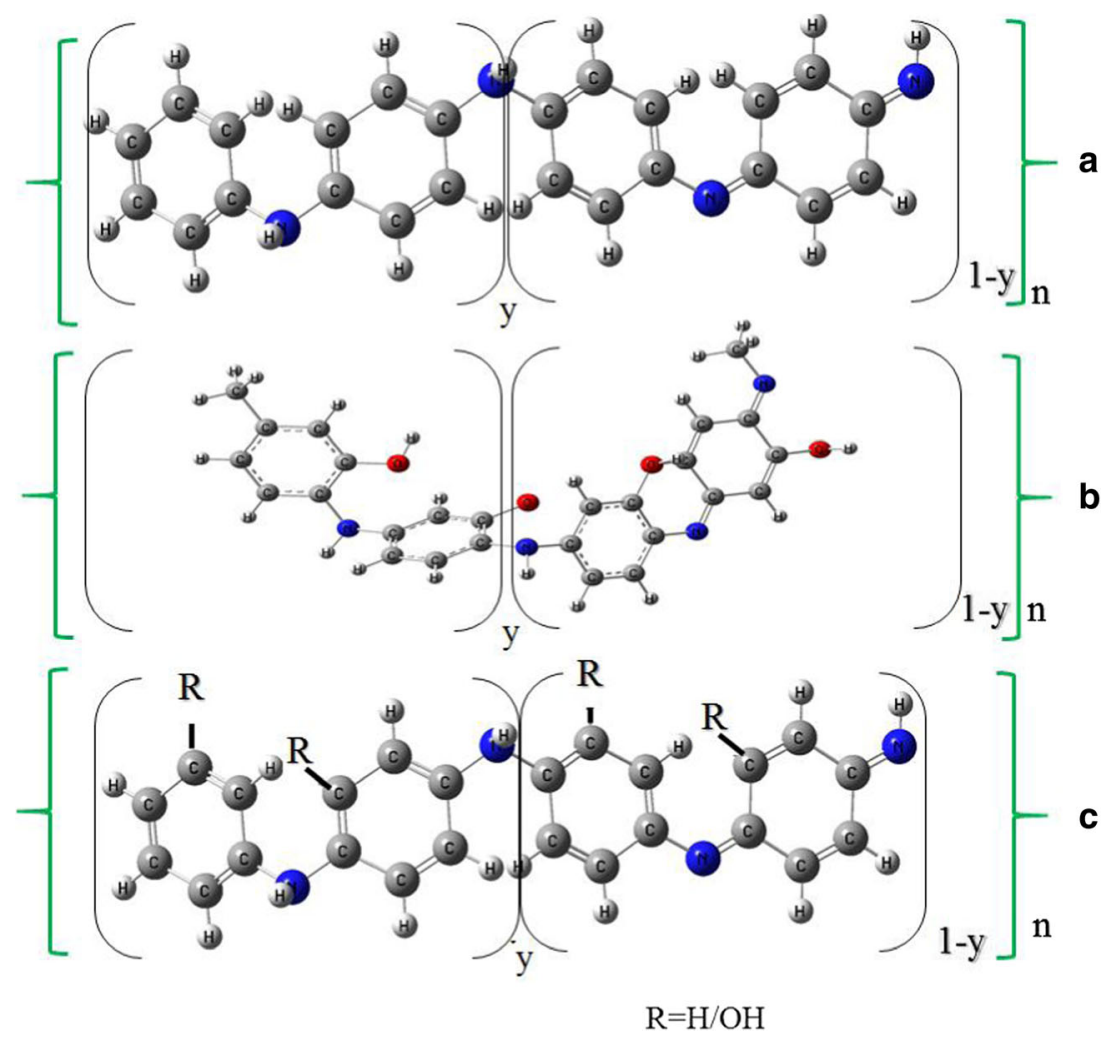

sonicated for $5 \mathrm{~min}$. The modified electrode was prepared by a simple drop-casting method, by pipetting a volume of $10 \mu \mathrm{L}$ on the GCE and dried at room temperature under vacuum condition.

\section{Characterization}

\section{Cyclic voltammetry}

All electrochemical tests were performed on a laboratory potentiostat (Gamry 3000, USA) with a glassy carbon electrode (GCE, $d=3 \mathrm{~mm}$, Bioanalytical systems, USA) used as a working electrode. $\mathrm{Ag} / \mathrm{AgCl} / 3 \mathrm{M} \mathrm{KCl}$ electrode and $\mathrm{Pt}$ electrode (Bioanalytical systems, USA) were applied in a threeelectrode cell system as reference and counter electrodes respectively. Cyclic voltammetric measurements were acquired under Gamry Instruments Framework software, and data acquired were evaluated using Echem Analyst and OriginPro 8.

Cyclic voltammetry test was conducted in $0.1 \mathrm{M} \mathrm{H}_{2} \mathrm{SO}_{4}$ acid solution as the electrolyte under $\mathrm{N}_{2}$ atmosphere, in the potential range from -0.4 to $0.8 \mathrm{~V}$ at a scan rate of $100 \mathrm{mVs}^{-1}$. Scan rate influence was also studied under similar conditions except for, scan rates varied at $10,20,30,40$, and $50 \mathrm{mVs}^{-1}$ in the potential range between -0.2 and $0.7 \mathrm{~V}$.

\section{XPS studies}

The XPS signals on the powdered sample were recorded using AXIS ULTRA DLD (Kratos Analytical Ltd., UK) equipped with an Al Ka X-ray source. The spectra were acquired in the constant analyzer energy mode with pass energy of $160 \mathrm{eV}$, $10 \mathrm{kV}$, and $10 \mathrm{~mA}$ emission current for the survey. The individual scans were performed with pass energy of $10 \mathrm{eV}$, $15 \mathrm{kV}$, and $15 \mathrm{~mA}$ emissions current. The Vision Manager 2 software was used for digital acquisition and data processing. Spectral calibration was determined using the automated calibration routine and the internal C1s standard. Scanning electron micrographs are taken with JEOL-6700 field emission microscope to study the morphology of the polymers.

In $100 \mathrm{ml}$ of DMF solvent, the solubility of PA and PA-coo-HA derivatives in the powder form is to be determined by dissolving $10 \mathrm{mg}$ of the polymer and allowed to disperse homogeneously. The dispersion is aged for few hours at the room temperature of $25^{\circ} \mathrm{C}$; the polymer dispersion is now being filtered through sintered glass crucible (porosity $2 \mu \mathrm{m}$ ).

\section{UV-Visible}

UV-Visible absorption spectra of the polymer samples in NMP solvent were recorded at room temperature on a 
Thermo Spectronic Genesys2 Research Grade Spectrophotometer.

\section{Results and discussion}

\section{Solubility test}

Processability plays an important role in conducting polymers and their applications. The solubility of PA, P-o-HA, and the copolymers dissolved individually in THF solvent and were presented in Table 1. The data reveals that the solubility of conventional PA $\left(8.78 \times 10^{-2}(\mathrm{~W} / \mathrm{V} \%),(\mathrm{g} / \mathrm{dL})\right)$ is lesser than the P-o-HA, and the copolymers. The homopolymer (P-oHA) solubility is better than ten times $\left(90.78 \times 10^{-2}(\mathrm{~W} / \mathrm{V}\right.$ $\%),(\mathrm{g} / \mathrm{dL}))$ to PA. However, the copolymer solubility increases gradually with increasing the weight percentage of ortho hydroxy aniline units in the polymer backbone and attained the maximum solubility $\left(73.35 \times 10^{-2}\right.$ to $87.56 \times$ $\left.10^{-2}(\mathrm{~W} / \mathrm{V} \%),(\mathrm{g} / \mathrm{dL})\right)$. In conclusion, the solubility of the PA is lesser than that of P-o-HA and copolymers, and the solubility ranges of the copolymers were in between the PA and homopolymer (P-o-HA). P-o-HA and copolymers were attaining remarkable solubility due to the presence of $-\mathrm{OH}$ (hydrogen bonding) group onto the polymer backbone, and which provide the more polar nature to the copolymer.

\section{Morphological properties}

The FE-SEM images of synthesized PA and PA-co-o-HA copolymer of magnification $80 \mathrm{k}$ are depicted in Fig. 1. The average particle size of the PA and copolymer is around 0.5 to $1 \mu \mathrm{m}$. The microscopy revealed rough topology with clusters surfacing on the top for the as-prepared PA (Fig. 1a). Interesting morphological changes occur in case of PA-co-oHA copolymer. Regular and perfectly shaped cabbage flower globules are developed (Fig. 1b). These globules formation could have taken place because of the hydrophobic nature of polyaniline particles and severe stirring conditions which catered the doped polymer's transformation into a globular structure.

Table 1 Solubility data of both homopolymers and copolymers

\begin{tabular}{ll}
\hline Polymers derivatives HCl salt & Solubility $(\mathrm{W} / \mathrm{V} \%),(\mathrm{g} / \mathrm{dL}), \mathrm{THF}$ \\
\hline P-o-HA & $90.78 \times 10^{-2}$ \\
PA-co-o-HA20 & $73.35 \times 10^{-2}$ \\
PA-co-o-HA40 & $81.00 \times 10^{-2}$ \\
PA-co-o-HA60 & $85.96 \times 10^{-2}$ \\
PA-co-o-HA80 & $87.56 \times 10^{-2}$ \\
PA & $8.78 \times 10^{-2}$ \\
\hline
\end{tabular}

\section{Structural properties}

\section{UV-Vis absorption study}

The UV-Vis electronic absorption spectroscopy is an important tool for conducting polymers and their composites to recognizing the conducting and insulating states. The PA, P$\mathrm{o}-\mathrm{HA}$, and copolymers dissolved in NMP and spectra recorded at room temperature in the range of $250-900 \mathrm{~nm}$. The spectra were shown in Fig. S1 [supplementary material]. The PA, P-o-HA, and copolymers showed two visible absorption bands around 310 and $580 \mathrm{~nm}$ in NMP solvent [35]. The lower wavelength band is attributed to the $\pi-\pi^{*}$ transition of the conjugated ring systems and the higher wavelength band is assigned to the $n-\pi^{*}$ exciton transition between the highest occupied molecular orbital (HOMO) of the benzenoid ring and the lowest unoccupied molecular orbital (LUMO) in the quinoid ring (i.e., benzenoid to quinoid excitonic transition). The obtained data for PANI, P-o-HA, and copolymers were much closed with other researchers [36, 37]. Decreasing the intensity of lower band wavelength may be due to growing hydroxy branching in the polymer backbone, which lacks the $\pi-\pi^{*}$ conjugation in between phenyl rings.

\section{XPS study}

XPS was examined to identify chemical element presence and chemical character at particular elements. The survey spectra of PA, P-o-HA, and copolymer polymers reveal that presence of expected elements in all samples and it is in good agreement with feeding in samples (Fig. 2). Figure 3 shows the N1s spectra of all three samples and Fig. 3a represents the $\mathrm{N} 1 \mathrm{~s}$ spectra for polyaniline and the curve deconvoluted into three peaks with $399.1,400.8$, and $402.1 \mathrm{eV}$ corresponding to $\mathrm{C}-\mathrm{N}$, neutral/tertiary amine $(\mathrm{C}=\mathrm{N})$ and quaternary amine/ protonated imine $\left(\mathrm{C}=\mathrm{NH}^{+}\right)$respectively. Similarly, in Fig. 3b, c), the same pattern was observed for P-o-HA and copolymer at 398.2, 399.5, and $401.5 \mathrm{eV}$ and 398.8, 399.7, and $402.3 \mathrm{eV}$ respectively. The obtained data for $\mathrm{N} 1 \mathrm{~s}$ for all the three above samples were close to literature. The binding energies for C1s spectra of PA, P-o-HA, and copolymer observed at $\sim 284.2,285.8,286.3$, and $287.6 \mathrm{eV}$ ascribed to $\mathrm{C}-\mathrm{C}$, $\mathrm{C}=\mathrm{N}, \mathrm{C}=\mathrm{NH}^{+}$, and $\mathrm{C}=\mathrm{O}$ functionality, respectively (Fig. $4 \mathrm{a}-$ c). The first integral detected at $\sim 284.2 \mathrm{eV}$ is attributed to the neutral $\mathrm{C}-\mathrm{C}$ bonds in the polymer backbone. The second component at $\sim 285.8 \mathrm{eV}$ can be consigned to the carbon bonded with neutral nitrogen atoms $(\mathrm{C}=\mathrm{N})$. Third peak can be ascribed to the carbon atoms bonded to charged nitrogen atoms $\left(\mathrm{C}=\mathrm{NH}^{+}\right)$. The highest binding energy $\sim 287 \mathrm{eV}$ is characteristic peak for the carbon connected with oxygen $(\mathrm{C}=\mathrm{O})$, and the $\mathrm{C}=\mathrm{O}$ functional groups are assigned to be present in PANI, P-o-HA, and copolymer due to the formation of benzoquinone (BQ) and hydroquinone (HQ) as degradation 
Fig. 1 SEM images of $\mathbf{a}$ PA and $\mathbf{b}$ PA-co-o-HA copolymer

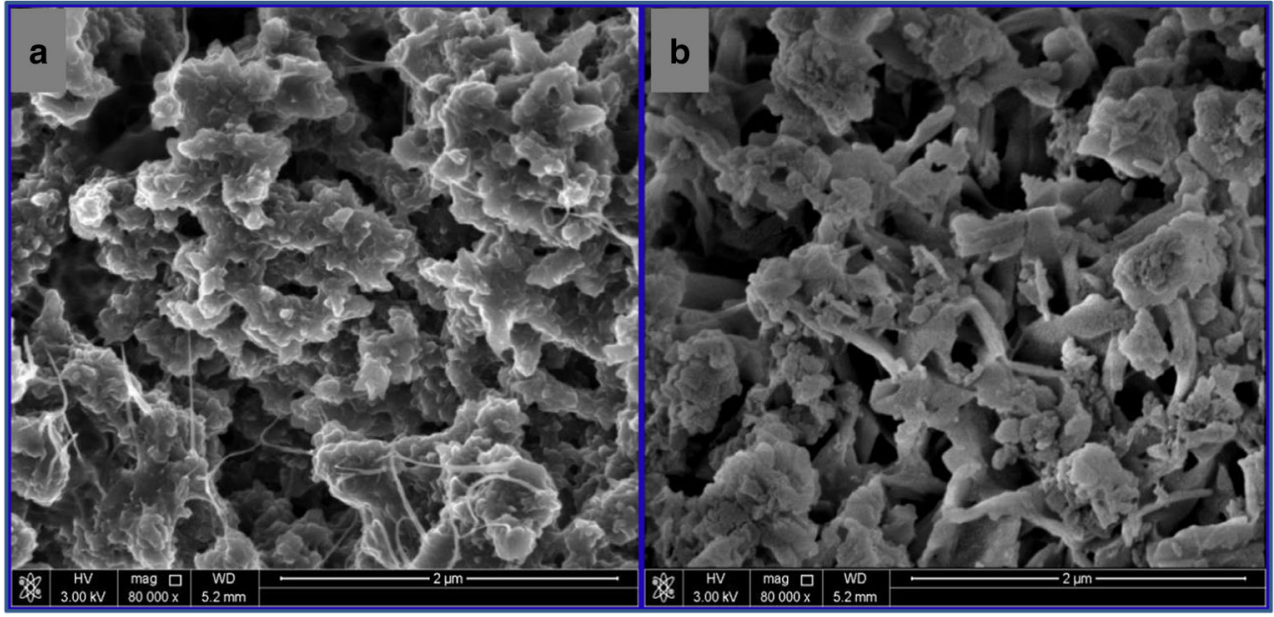

products [38]. The O1s spectra were not considered for further analysis of synthesized polymers since it is extremely influenced by moisture and oxide residue impurities that are common for XPS analysis. Finally, this XPS data also suggests that the formation PA, P-o-HA, and copolymer in emeraldine salt (ES) form [39].

\section{Electrochemical performance}

The electrical conductivity and electrochemical properties are most significant properties for the conducting polyaniline. The as-prepared PA, P-o-HA, and copolymers were investigated for their electrochemical activity by $\mathrm{CV}$ using $\mathrm{GCE}$ as a working electrode in a three-electrode system. The electrochemical tests were performed in $0.1 \mathrm{M} \mathrm{H}_{2} \mathrm{SO}_{4}$ acid solution as the electrolyte under $\mathrm{N}_{2}$ inert atmosphere, in the potential window from -0.4 to $0.8 \mathrm{~V}$ at different scan rates ranging from 20 to $50 \mathrm{mV} \mathrm{mVs}^{-1}$. As shown in Fig. 5, the conventional polyaniline (PA) shows two forward peaks at 0.286 (peak 1) and $0.540 \mathrm{~V}$ (peak 2) and two backward (reverse) peaks at 0.286 and $0.540 \mathrm{~V}$ corresponding to two singleelectron oxidations and two-single electron reductions reversibly at different scan rates. The similar pattern was observed in P-o-HA homopolymer as shown in Fig. 5a. Similarly, the first and second oxidation peaks for copolymer are observed at $0.246 \mathrm{~V}$ and $0.445 \mathrm{~V}$, very close to PA and P-o-HA. The peak
Fig. 2 XPS survey for a PA, b Po-HA, c copolymer
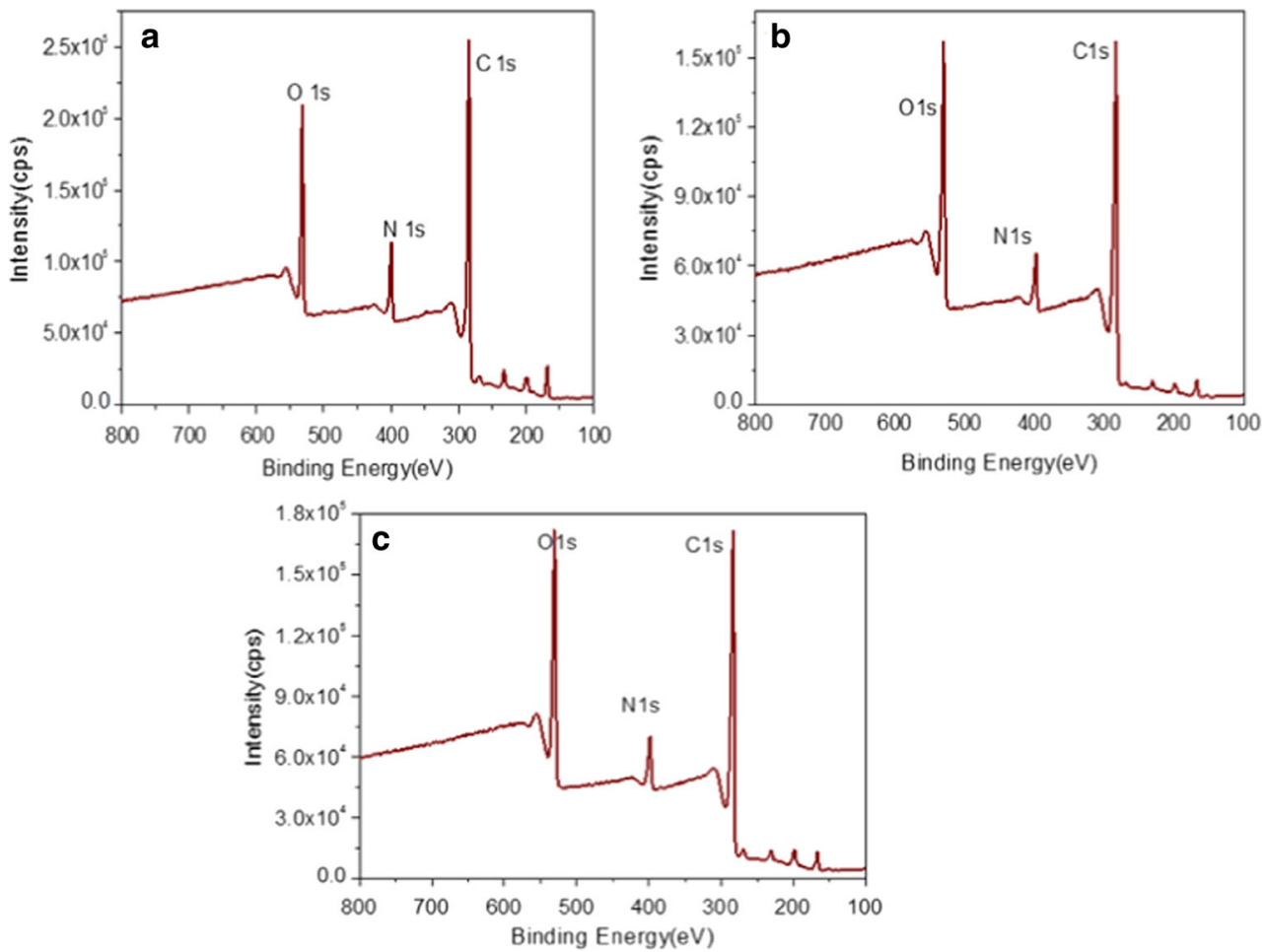
Fig. 3 XPS spectra for $\mathrm{N} 1 \mathrm{~s}$ region for a PA, b P-o-HA, c copolymer

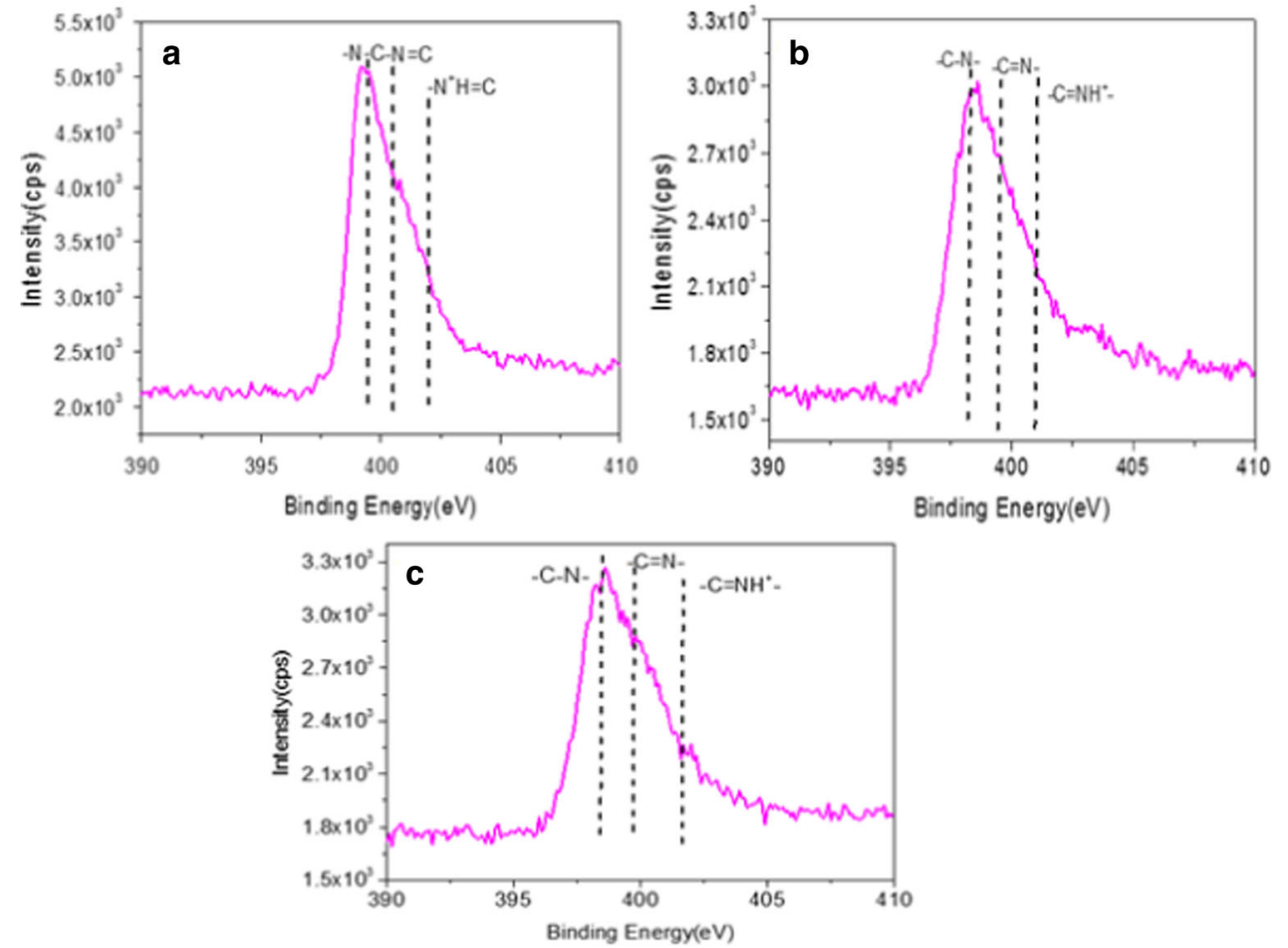

current of PA is higher compared to P-o-HA and which in turn is higher when compared to the copolymer. This is due to increasing the hydroxyl (-OH) branching on the polymer backbone in the polymer chain. In general, while branching increases in copolymers the planarity of the phenyl ring will be reduced, this leads to the decreasing in conjugation and conductivity as well. The current density decreases from PAco-o-HA20 to PA-co-o-HA80 by increasing the weight percentage of o-hydroxyaniline in the polymeric backbone. The oxidation peaks correspond to the transitions from
Fig. 4 XPS spectra for $\mathrm{C} 1 \mathrm{~s}$ region for a PA, b P-o-HA, c copolymer
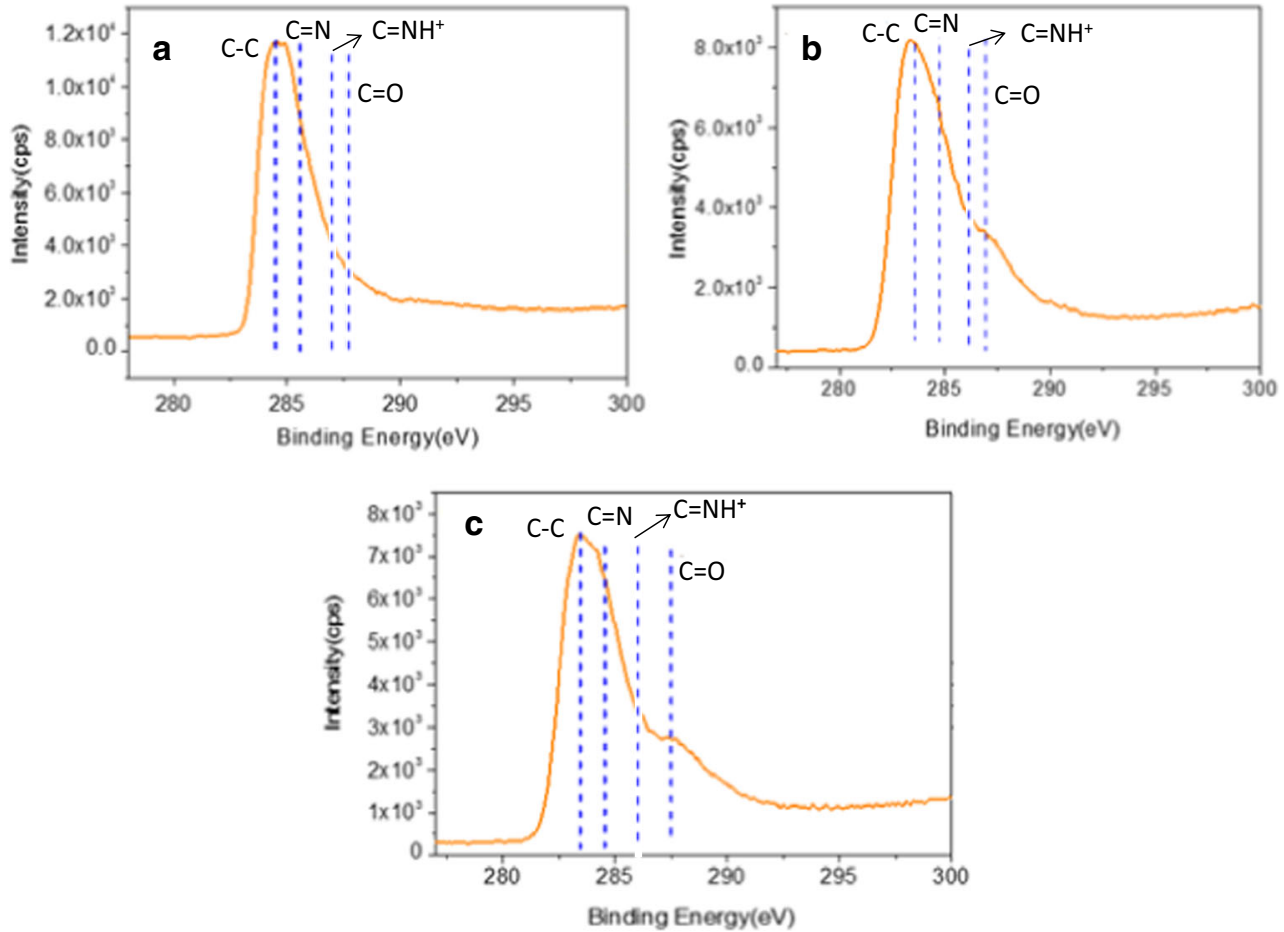
Fig. 5 Cyclic voltammetry scan of a P-o-HA, b PA, c PA-co-o-HA 80, d PA-co-o-HA 20
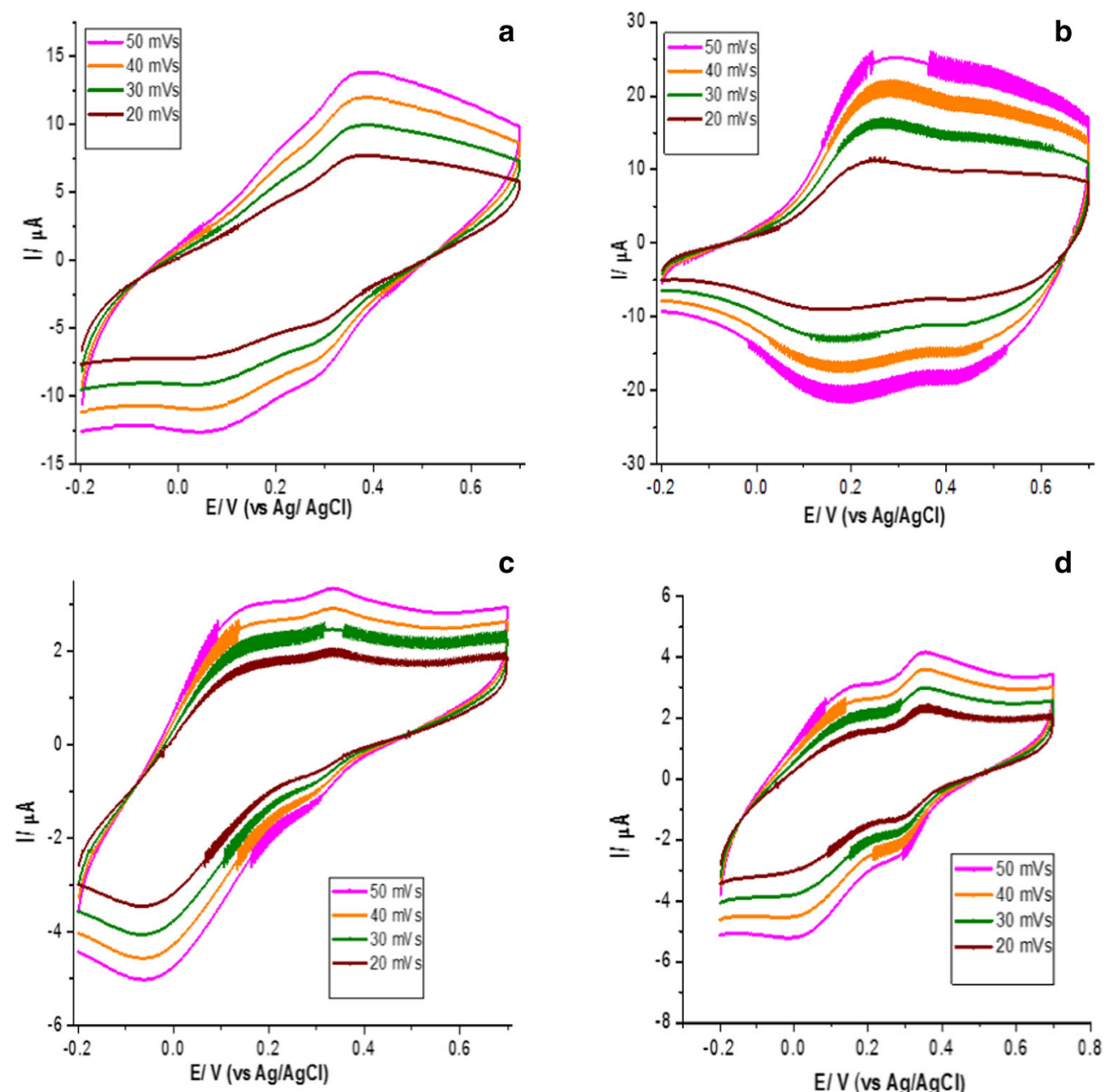

C

a

d

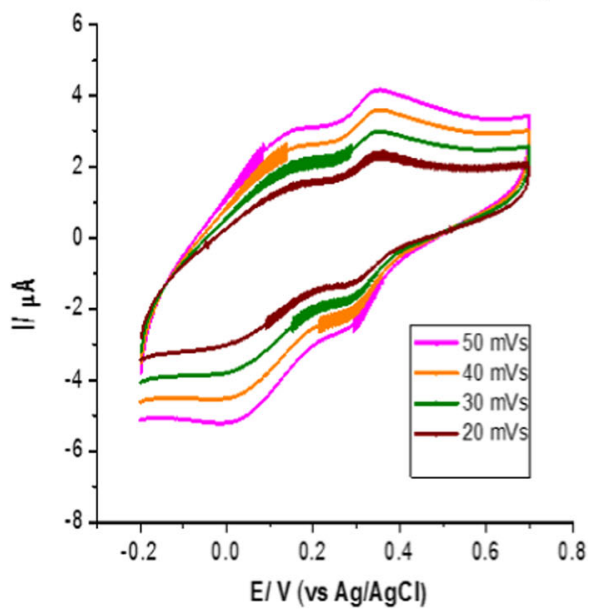

leucoemeraldine base (LEB) to emeraldine base (EB) oxidation state and EB to pernigraniline (PA) oxidation state, respectively.

\section{Conclusions}

In summary, using a chemical oxidative polymerization, we have successfully synthesized poly(aniline-co-ohydroxyaniline) copolymers with varying the amount of ohydroxyaniline $(20,40,60$, and $80 \%)$ and named as PA-coo-HA20, PA-co-o-HA40, PA-co-o-HA60, and PA-co-o-HA80 respectively. The possible formation of polymers and copolymer structures were explained in detail with the help of FESEM, UV, and XPS analysis. The solubility of PA, P-o-HA, and the copolymers dissolved individually in THF solvent and the solubility of conventional PA $\left(8.78 \times 10^{-2}\right.$ (W/V \%), $(\mathrm{g} / \mathrm{dL}))$ is lesser than the P-o-HA, and the copolymers. The homopolymer (P-o-HA) solubility is better than ten times to PA. However, the copolymer solubility increases gradually with increasing the weight percentage of ortho hydroxy aniline units in the polymer backbone and attained the maximum solubility $\left(74.88 \times 10^{-2}\right.$ to $\left.87.56 \times 10^{-2}(\mathrm{~W} / \mathrm{V} \%),(\mathrm{g} / \mathrm{dL})\right)$. From cyclic voltammograms, the peak current of $\mathrm{PA}$ is higher compared to P-o-HA and which in turn is higher when compared to copolymer and the current density decreases from PA-co-o-HA20 to PA-co-o-HA80 by increasing the weight percentage of o-hydroxyaniline in polymeric backbone.

Acknowledgements We gratefully acknowledge the Qatar University, Doha, for providing necessary research funding. We also acknowledge the instrumentation facilities provided at CLU and CAM of Qatar University, Doha.

Open Access This article is distributed under the terms of the Creative Commons Attribution 4.0 International License (http:// creativecommons.org/licenses/by/4.0/), which permits unrestricted use, distribution, and reproduction in any medium, provided you give appropriate credit to the original author(s) and the source, provide a link to the Creative Commons license, and indicate if changes were made. 


\section{References}

1. Wang H, Jianyi L, Xiang Shen Z (2016) J Sci Adv Mater Devices 1: 225-255

2. Sangodkar H, Sukeerthi S, Srinivasa RS, Lal R, Contractor AQ (1996) Anal Chem 68

3. Langer JJ, Langer RK (2005) Rev. Adv Mater Sci 10:434

4. Bhadra S, Khastgir D, Singha NK, Lee JH (2009) Prog Polym Sci $34: 783 \mathrm{e} 810$

5. Lu WK, Elsenbaumer RL, Wessling B (1995) Synth Met 71:2163

6. Ahmad N, MacDiarmid AG (1996) Synth Met 78:103

7. Ravi A, Mohanraj V, Kim AR, Yoo DJ (2019) J Alloys Compd 771: 477-488

8. Wang YZ, Gebler DD, Lin LB, Blatchford JW, Jessen SW, Wang HL, Epstein AJ (1996) Appl Phys Lett 68:894

9. Chen SA, Chuang KR, Chao CI, Lee HT (1996) Synth Met 82:207

10. MacDiarmid AG, Yang LS, Huang WS, Humphrey BD (1987) Synth Met 18:393

11. Kang ET, Neoh KG, Tan KL (1992) Surf Interface Anal 19:33

12. MacDiarmid AG, Chiang JC, Richter AF, Epstein AJ (1987) Synth Met 18:285

13. Kinlen PJ, Frushour BG, Ding Y, Menon V (1999) Synth Met 101: 758-761

14. Skotheim TA (1986) Handbook of conducting polymers, vol 1. Marcel Dekker, New York, $p$ and 2

15. Trivedi DC, Nalwa HS (1997) Handbook of organic conductive molecules and polymers, vol 2. Wiley, Chichester

16. Park SM, Nalwa HS (1997) Handbook of organic conductive molecules and polymers, vol 3. Wiley, Chichester

17. Macinnes D, Funt BL (1988) Synth Met 25:235-242

18. Gazotti WA Jr, de Paoli MA (1996) Synth Met 80:263-269

19. Yue J, Wang ZH, Cromack KR, Epstein AJ, MacDiarmid AG (1991) J Am Chem Soc 113:2665-2671

20. Wei XL, Wang YZ, Long SM, Bobeczko C, Epstein AJ (1996) J Am Chem Soc 118:2545-2555
21. Dalas E (1992) J Mater Sci 27:453-457

22. Waware US, Summers GJ, Mohd R, Hamouda AMS (2017) Ionics. 24:118

23. Clioi HJ, Kim JW, To K (1999) Synth Met 101:697-698

24. McManus PM, Cushman RJ, Yang SC (1987) J Phys Chem 91:744

25. Stafstrm S, Das JL, Epstein AJ, Woo HS, Tanner DB, Huang WS, MacDiarmid AG (1987) Phys Rev Lett 59:1464

26. Kaya M, Kitani A, Sasaki K (1986) Chem Lett:147

27. Stafstrm S, SjSgren B, Wennerstr SO, Hjertberg T (1986) Synth Met 16:31

28. Genies EM, Lapkowski MJ (1987) Electroanal Chem 220:67

29. Furukawa Y, Hara T, Hyodo Y, Harada I (1986) Synth Met 16:189

30. Ohira M, Sakai T, Takeuchi M, Kobayashi Y, Tsuji M (1987) Synth Met 18:347

31. Regina M, Gediminas N, Albertas M (2019) Synth. Met, vol 248, pp 35-44

32. Chiang JC, MacDiarmid AG (1986) Synth Met 13:193

33. Baughman RH, Wolf JF, Eckhardt H, Shacklette LW (1988) Synth Met 25:121

34. Azmi R, Trouillet V, Strafela M, Ulrich S, Ehrenberg H, Bruns M (2017) Surf Interface Anal:1-9

35. Yang Z, Wang X, Yang Y, Liao Y, Wei Y, Xie X (2010) Langmuir 26:9386-9392

36. Jing X, Wang Y, Wu D, Qiang J (2007) Ultrason Sonochem 14:7580

37. Manohar SK, MacDiarmid AG (1991) Synth Met 41:711-714

38. Sebastian G, Anna K, Mats F, Krzysztof L, Jerzy JL (2008) Solid State Ionics 179:2234-2239

39. Rohit RG, Amir HFN, Marlies H, Debra JS, Nanjundan AK, Zhao XS (2017) J Mater Chem A 5:22186-22192

Publisher's note Springer Nature remains neutral with regard to jurisdictional claims in published maps and institutional affiliations. 